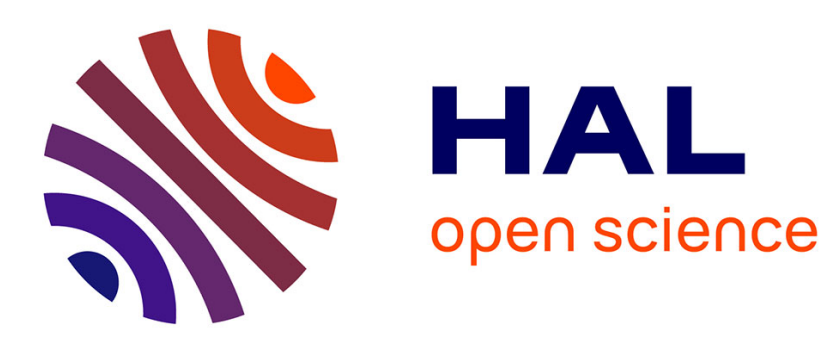

\title{
Les associations d'arbres et de cultures intercalaires annuelles sous climat tempéré
}

\author{
Christian Dupraz
}

\section{To cite this version:}

Christian Dupraz. Les associations d'arbres et de cultures intercalaires annuelles sous climat tempéré. Revue forestière française, 1994, 46 (S), pp.72-83. 10.4267/2042/26619 . hal-03444484

\author{
HAL Id: hal-03444484 \\ https://hal.science/hal-03444484
}

Submitted on 23 Nov 2021

HAL is a multi-disciplinary open access archive for the deposit and dissemination of scientific research documents, whether they are published or not. The documents may come from teaching and research institutions in France or abroad, or from public or private research centers.
L'archive ouverte pluridisciplinaire HAL, est destinée au dépôt et à la diffusion de documents scientifiques de niveau recherche, publiés ou non, émanant des établissements d'enseignement et de recherche français ou étrangers, des laboratoires publics ou privés. 


\title{
LES ASSOCIATIONS D'ARBRES ET DE CULTURES INTERCALAIRES ANNUELLES SOUS CLIMAT TEMPÉRÉ
}

\author{
C. DUPRAZ
}

L'histoire de notre agriculture est celle d'un long processus de défrichement, d'une lutte contre la forêt. Jusqu'au XIV siècle en Europe, les parcelles agricoles étaient issues de la forêt, et conservaient de très nombreux arbres, gardés pour leurs fonctions productives ou symboliques. Progressivement les arbres seront extirpés des parcelles cultivées. Avec l'introduction des araires et la généralisation de l'emploi de la traction animale, les arbres deviendront des obstacles. Avec la mécanisation, ils seront systématiquement supprimés.

En cette fin de $X X^{e}$ siècle, ce patrimoine séculaire de sols défrichés est remis en cause. Sa fonction productive de biens alimentaires n'est plus aussi prioritaire en Europe. Des programmes de jachère obligatoire sont élaborés, pour réduire la production agricole tout en conservant le patrimoine de sols cultivés intact, ce qui permettra une remise en culture à tout moment. Des boisements de terres agricoles sont mis en cuvre, pour réduire irréversiblement les surfaces de terres arables. Et parfois, les propriétaires fonciers renoncent à gérer leur patrimoine, acceptant la friche, et plus tard un boisement spontané peu productif, souvent fragile devant les incendies. Ce processus a changé de nature depuis une dizaine d'années, car il concerne désormais de plus en plus des terres agricoles de qualité.

Alors, jachère, friche ou boisement, pas d'alternatives ? Et pourquoi pas l'agroforesterie ?

L'association d'arbres et de peuplements herbacés fourragers est, avec les brise-vent, la forme d'agroforesterie la plus répanđue dans les zones tempérées du globe. Ces peuplements intercalaires fourragers peuvent être spontanés, enrichis par sursemis, ou semés. Mais leur caractéristique essentielle, du point de vue de la gestion de l'association arbres - herbacées, est leur pérennité. De ce fait, les interventions de travail du sol sont relativement limitées. La principale difficulté consiste à faire coexister les arbres avec les animaux (Dupraz et al., 1993) lorsqu'il y a pâturage direct.

Lorsque les cultures intercalaires sont des plantes annuelles, les contraintes sont différentes. Les interventions de travail du sol, de fertilisation, de protection phytosanitaire, de récolte sont beaucoup plus fréquentes, mais la protection de l'arbre est moins cruciale qu'en présence d'animaux. La gestion simultanée des arbres et des cultures annuelles intercalaires exige une grande technicité.

Nous nous proposons, dans cet article, de faire une synthèse des connaissances sur l'agroforesterie tempérée avec cultures intercalaires annuelles, qu'il convient de dénommer agrisylviculture pour être en conformité avec la nomenclature internationale. Dans un second article, nous tenterons ensuite d'imaginer les perspectives de développement d'une agroforesterie dans les exploitations qui pratiquent des cultures annuelles (céréales, oléoprotéagineux, tubercules) en Europe. 


\section{L'AGROFORESTERIE CHINOISE EN ZONE TEMPÉRÉE}

L'agroforesterie chinoise est considérée par les agronomes chinois comme un cas particulier d'agriculture écologique (Saint-Pierre, 1994). Cette agriculture écologique est présentée comme la voie chinoise vers le progrès agricole, sans faire le détour par l'intensification à base d'intrants sur le modèle des pays occidentaux. Elle se situe dans une logique d'intensification de l'utilisation de l'espace et fait appel à une quantité impressionnante de main-d'œuvre.

\section{Une grande diversité d'associations arbres - cultures}

Deux modèles agroforestiers ont connu un développement spectaculaire au cours des vingt dernières années en Chine. II s'agit des associations Paulownias - céréales en zone tempérée et hévéa - thé en zone tropicale.

Mais bien d'autres associations agroforestières sont également utilisées en zone tempérée (Zhu et al., 1991a) : de nombreuses espèces d'arbres comme les Jujubiers fruitiers (Ziziphus jujuba), les Peupliers (Populus tomentosa, fastigata, deltoides) et les Frênes (Fraxinus sinensis) sont associées à diverses cultures intercalaires (principalement des céréales et des oléoprotéagineux : blé tendre, maïs, colza, soja).

Bien que les auteurs chinois soulignent l'importance des cultures mixtes dans la Chine ancienne (You, 1991), toutes ces pratiques agroforestières sont récentes et, il faut le souligner, résultent de travaux de recherches. Les agroforesteries traditionnelles n'utilisaient pas les essences d'arbres qui sont préconisées aujourd'hui. Les arbres les plus fréquemment cités dans les descriptions de systèmes agroforestiers pratiqués il y a 2000 ans sont le Sophora, les Mûriers, le Broussonetia, certains Cunninghamia (You, 1991).

En particulier, l'agroforesterie du Paulownia, qui représente selon les sources de 1,3 (Zhu et al., 1991a) à 3 (He, 1991) millions d'hectares dans les grandes plaines du Nord de la Chine, est une pratique récente, encore inconnue il y a trente ans. De même, l'agroforesterie de l'Hévéa, qui a fait l'objet d'études poussées dans les années 70, n'a été adoptée que récemment, sur l'île de Hainan, hors de la zone pour laquelle elle avait été mise au point (Saint-Pierre, 1994).

\section{L'agroforesterie du Paulownia}

Comme pour le sylvopastoralisme du Pin radiata en Nouvelle-Zélande (Dupraz et al., 1992), le succès de l'agroforesterie du Paulownia doit beaucoup à l'amélioration génétique des arbres utilisés (Zhu et al., 1991b). L'Académie forestière chinoise (AFC) poursuit depuis 1973 un programme d'amélioration génétique du Paulownia (AFC, 1985). Avec l'aide de I'IDRC (Canada), l'AFC a mis en place les principales expérimentations d'agroforesterie du Paulownia, notamment dans la région de Dang Shan, située dans la province de Anhui. Ces expérimentations ont permis de préciser les densités optimales de plantations agroforestières. L'AFC gère également 116 ha de tests de comparaison de clones et d'évaluation de descendances. La croissance exceptionnellement rapide du Paulownia permet d'abtenir couramment des billes d'un diamètre de $25 \mathrm{~cm}$ en une dizaine d'années et, dans les meilleures conditions, de $40 \mathrm{~cm}$ en 10 ans (AFC, 1985). Les révolutions les plus fréquentes durent une quinzaine d'années.

Les ouvrages chinois indiquent une telle palette d'utilisations du bois de Paulownia qu'il est nécessaire de faire le tri entre ce qui peut être considéré comme anecdotique, et ce qui a vraiment un poids économique. Le bois de Paulownia se distingue par sa densité très faible, et surtout par l'absence presque complète de déformation au séchage. Il est extrêmement facile à travailler et à polir. II est très utilisé pour fabriquer des meubles, et de nombreux objets de petite taille. Son utilisation industrielle de l'avenir est sans conteste le contreplaqué, pour lequel plusieurs usines chinoises ont démarré des productions à grande échelle. Le Japon importe toutes les grumes de 


\section{DUPRAZ}

Paulownia disponibles sur le marché mondial, car ce bois y est très recherché. Pour l'anecdote, signalons que les cartes de visite des hommes d'affaire japonais sont très souvent en ... bois de Paulownia. Les feuilles de Paulownia sont partout ramassées au sol en automne et utilisées comme fourrage, et se vendent souvent sur les marchés, attachées en gros paquets. Curieusement, l'utilisation en bois de chauffage des branches n'est pas signalée dans la littérature, peut-être parce que cela va de soi?

Les deux espèces de Paulownia les plus intéressantes pour l'agroforesterie sont Paulownia elongata et Paulownia fortunei. Un hybride P. elongata $\times P$. fortunei est maintenant largement utilisé (Henan Hybrid Number One, Yu Za Yi Hao). II serait résistant aux mycoplasmes (balais de sorcière), qui sont la principale pathologie du Paulownia en Chine. Un nouvel hybride très résistant à cette maladie est actuellement en cours de test (Mau-Bai 33, hybride de tomentosa et fortunei). Six clones à croissance rapide et relativement résistants aux maladies sont principalement utilisés dans les plantations agroforestières : trois clones de P. fortunei (C001, C020, PS01), un clone de $P$. tomentosa (C161), un clone de P. elongata (C125), et un clone hybride (PH01). 20 millions de plants issus de ces clones ont été mis en place ces dernières années (Zhu et al., 1991b). Aucun de ces clones n'a, à notre connaissance, été introduit en Europe, où la plupart des Paulownias introduits sont des $P$. tomentosa (= imperialis) issus de graine. Cette espèce est considérée par les forestiers chinois comme la moins intéressante pour l'agroforesterie, compte tenu de sa croissance (relativement) lente et de sa forme souvent défectueuse.

Les trois principales rotations de cultures intercalaires dans les plantations de Paulownia sont les suivantes : blé tendre et coton, blé tendre et sorgho, blé tendre - maïs - haricot. Elles représentent près de $90 \%$ des cultures intercalaires sous Paulownia. Plus de $70 \%$ des plantations de Paulownia se font à des espacements de $5 \times 20$ et $5 \times 40 \mathrm{~m}$, soit 100 et 50 arbres/ha. Les arbres sont coupés en fonction des besoins de trésorerie de la famille de l'exploitant, en général vers l'âge de dix ans. Dans la plupart des cas, les premières plantations datent des années 70 , et nous en sommes souvent maintenant à la troisième révolution.

Les densités de plantation ont fait l'objet de nombreux travaux expérimentaux et de simulations économiques (He, 1991). À partir de calculs de valeurs actualisées nettes (avec un taux de $8 \%$ ) à l'échelle de la parcelle, cet auteur montre que, d'un point de vue économique, les arbres représentent la majeure partie du revenu d'une parcelle agroforestière. Ceci conduirait, en situation de marché bien approvisionné en produits vivriers, à donner la préférence à de fortes densités d'arbres ( $5 \times 6$, soit 333 arbres/ha), et à des récoltes des arbres relativement tardives (vers 12 ans). Mais la nécessité pour les exploitants d'assurer leur indépendance vivrière conduit à modifier la procédure d'optimisation, pour tenir compte de la nécessité impérieuse de récolte d'une quantité minimale de céréales par famille (250 kg de céréales/personne/an). On montre alors que l'optimum se situe effectivement à des espacements de $5 \times 40 \mathrm{~m}$ pour des récoltes des Paulownias à 10 ou 11 ans. Ce schéma de plantation permet sur l'ensemble de la révolution de produire $90 \%$ de la production vivrière que l'on obtiendrait sur une parcelle sans arbres, et correspond bien aux pratiques actuellement observees chez les paysans.

Ces plantations équiennes à densité définitive sont également comparées à des plantations avec éclaircies, et à des plantations à deux strates arborées d'âges différents. Dans le premier cas, une plantation à 200 arbres/ha est ramenée à 100 arbres/ha à 6 ans, puis à 50 arbres/ha à 8 ans, avant une coupe finale à 11 ans. Dans le second cas, la densité reste de $100 \mathrm{arbres} / \mathrm{ha}$, mais avec deux générations d'arbres décalées de 6 ans, et des coupes à 11 ans. Des études de sensibilité ont montré que ce dernier modèle est le plus intéressant, car le moins sensible à des fluctuations des coûts et des prix des produits. II est donc présenté comme l'optimum absolu, à adopter sans réserve. Compte tenu du mode très empirique de pondération des objectifs dans le calcul, on peut critiquer la méthode, mais la force de ces résultats est qu'ils s'appuient sur des données réelles, obtenues en de nombreux sites, et à tous les stades du cycle du Paulownia. Même en agroforeste- 


\section{L'agroligniculture}

rie, les excès du centralisme chinois sont étonnants: on n'hésite pas à identifier LE modèle optimum unique, pour l'ensemble des paysans de l'immense zone climatique concernée!

La convivialité du Paulownia avec les cultures intercalaires est proverbiale. Cela est dû à des caractéristiques particulières de l'arbre: foliaison tardive, alors que les cultures intercalaires sont au stade remplissage du grain (colza) ou montaison (blé), enracinement profond peu en contact avec celui des cultures intercalaires (figure 1, ci-contre), ombre portée peu dense. Mais cela est surtout permis par le régime de pluies d'été qui réduit les risques de compétition pour l'eau entre les arbres et les cultures intercalaires (Wu et Dalmacio, 1991). Cette caractéristique, évidemment non transposable à l'Europe de l'Ouest, illustre une limite fondamentale du modèle chinois.

Figure 1

DISTRIBUTION VERTICALE

DE L'ENRACINEMENT DE PAULOWNIAS

(moyenne sur 3 arbres de 9,11 et 12 ans)

Dessiné d'aprés les données de CAF, 1986

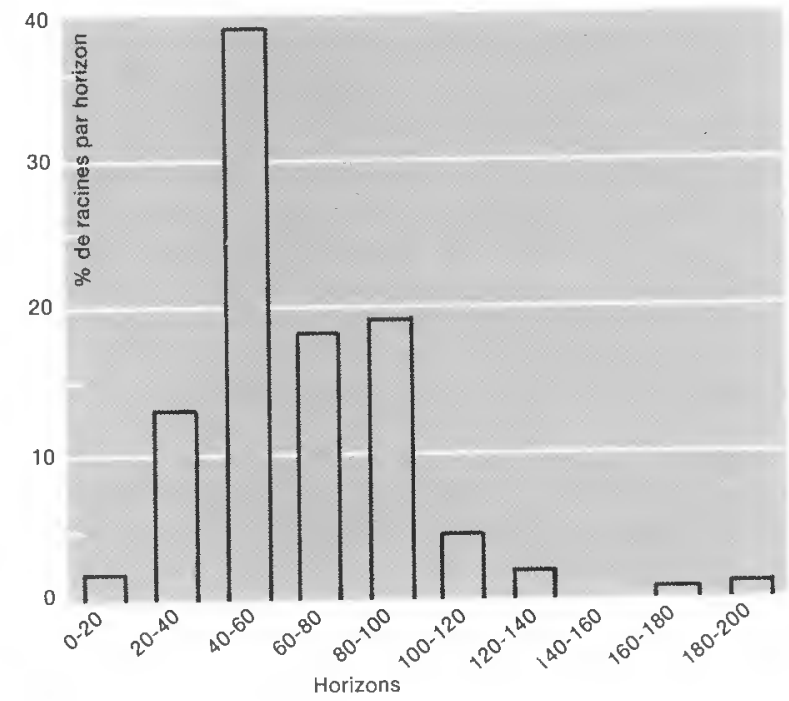

Les céréales de printemps souffrent souvent en Chine de l'effet desséchant des vents brûlants de début d'été. Par sa structure de brise-vent diffus alvéolaire, le peuplement de Paulownia protège les cultures intercalaires. Dans les zones exposées, le rendement des céréales est nettement amélioré par la présence des arbres (Zhu et al., 1991).

\section{La voie chinoise vers l'agroforesterie}

Les fermes pilotes agroforestières que l'on fait traditionnellement visiter aux étrangers représentent des situations atypiques extrêmement subventionnées, sans grande signification du point de vue du développement. C'est notamment le cas des fermes agroforestières des zones marécageuses du Jiangsu (fermes de Ma Peng ou de Chang Xia, avec leurs célèbres plantations agroforestières de Taxodium ascendens, Taxodium distichum et Metasequoia glyptostroboides avec cultures intercalaires de colza, blé, soja, plantes fourragères, pâturage d'oies et de chèvres...). Ces fermes ont séduit plus d'un visiteur étranger par leur beauté et l'intelligence de leur conception (Étaix et Le Hir, 1988), mais restent très marginales.

En matière de recherche agroforestière, la Chine se distingue par les aspects suivants :

- une analyse méthodique de toutes les pratiques observables chez les paysans, même si ces dernières sont très marginales ou exceptionnelles;

- une approche parcellaire, qui néglige en général de comparer l'association de cultures et l'assolement de cultures pures. On ne trouve pratiquement pas de publications sur les coefficients de rendement équivalents qui permettent cette comparaison ;

- une approche monographique, avec des études de cas de très nombreuses associations agroforestières. Dans une vidéo récente sur l'agroforesterie chinoise, pas moins de 80 associations agroforestières sont filmées ; 


\section{DUPRAZ}

- une priorité donnée aux problèmes de compétition pour la lumière (Liu et al., 1991), ce qui résulte du climat de mousson à pluies extrêmement groupées pendant la principale saison de végétation.

Le succès de l'agroforesterie à base de Paulownia suscite d'ailleurs des compétitions très vives entre l'Académie forestière chinoise et l'Institut du Paulownia, qui est une émanation de certaines universités agricoles provinciales (notamment celle du Henan).

En matière de diffusion des pratiques agroforestières, on notera les aspects suivants.

Ce sont essentiellement les fermes d'État qui diffusent les modèles agroforestiers, à partir de décisions politiques venues du plus haut niveau. Cependant, l'introduction des arbres est bien vécue par les paysans, car les problèmes de propriété ont été pris en compte: les arbres sont parfaitement appropriés, chaque paysan est propriétaire des arbres situés sur ses terres (le principe général est que celui qui plante un arbre en est propriétaire, même s'il n'est pas propriétaire du sol). Par exemple, dans le district de Woyang (Province de Anhui), chaque agriculteur possède 3 à 5 Paulownias. On a même parfois redécoupé les lots individuels de terres en bandes orthogonales aux lignes de plantation des arbres de manière à répartir de façon égalitaire les arbres entre les agriculteurs (Chirko et al., 1993).

La lecture des rapports récents de référence sur l'agroforesterie du Paulownia (Zhu, 1991) suscite l'admiration et la perplexité. Les chercheurs semblent avoir tout étudié, des propriétés insecticides de la pâte de feuille de Paulownia jusqu'à la qualité protéique (améliorée) du blé cultivé sous des Paulownias, en passant par l'activité microbiologique du sol, ou la teneur en $\mathrm{CO}_{2}$ de l'air sous les arbres. L'effet rhétorique de l'énumération d'usages incompatibles est fréquemment utilisé : ainsi les feuilles mortes de Paulownia sont un excellent fourrage et un excellent engrais. Mais, dans la pratique, il faut bien choisir! Ce résultat est déjà étonnant, car en général les feuilles mortes d'arbres sont de piètres fourrages. Les auteurs chinois expliquent ce résultat par le fait que le Paulownia a une croissance indéfinie, arrêtée par les gels qui bloquent les processus de translocation des assimilats vers les tissus de réserve dans le bois. Notons également cette expérience typiquement chinoise, où la litière d'un hectare de Paulownias de 8 ans (à 100 arbres/ ha) a été ramassée et épandue et incorporée dans un champ sans Paulownia, ce qui représentait 3 tonnes de MS/ha. Le résultat a été une augmentation de $31 \%$ du rendement en blé et de $20 \%$ du rendement en coton, par rapport à une parcelle non fertilisée.

Les arguments favorables à l'agroforesterie du Paulownia sont donc extrêmement nombreux, et i) est difficile de trouver dans la littérature chinoise traduite en anglais des réflexions critiques sur ce sujet, ce qui peut laisser planer un doute. Il y a de toute évidence un discours politique sur l'agroforesterie en Chine. En fin de compte, il reste ces millions d'arbres plantés et récoltés chaque année par les paysans chinois dans leurs champs. Et là, le doute n'est pas possible.

\section{PRATIQUES TRADITIONNELLES D'AGROFORESTERIES TEMPÉRÉES AVEC CULTURES INTERCALAIRES ANNUELLES}

Aucune agroforesterie tempérée ne peut, à notre connaissance, rivaliser avec les pratiques agroforestières chinoises du point de vue de l'ampleur de la mise en œuvre chez les paysans. Pourtant, il existe d'autres systèmes agroforestiers avec cultures intercalaires annuelles dignes d'intérêt.

\section{Pratiques traditionnelles en Asie Centrale}

Dans le Nord de l'Inde, au Pakistan, au Népal, la culture d'arbres dans les champs cultivés est une pratique traditionnelle très vivante (Tejwani, 1987). Les cultures intercalaires sont essentiellement le 
blé, l'orge et les pommes de terre. Les essences d'arbres sont les suivantes: Grewia optiva, Celtis australis, Morus serrata, Moringa oleifera et divers Chênes. Les arbres ont toujours une double finalité bois - fourrage : ils sont régulièrement émondés pour nourrir les animaux, et ont donc une production à moyen terme (premier émondage à 10 ans environ). Ces systèmes de culture sont entièrement manuels et chaque exploitant possède en moyenne une trentaine d'arbres disséminés sur son hectare. Aucune référence technique ou économique sur ces pratiques ne semble disponible.

Des expérimentations récentes d'agroforesterie avec le Peuplier (Populus deltoides) sont en cours à I'Institut de Recherches forestières de Dehra Duhn en Uttar Pradesh dans le Nord de I'Inde (Tewari, 1993). La principale culture intercalaire est le blé d'hiver, et les densités d'arbres restent très élevées. Les arbres sont cependant coupés à 5 ans, pour la fabrication d'allumettes, et les cultures intercalaires sont possibles les trois premières années. Ces schémas agroforestiers sont donc caractérisés par des révolutions extrêmement courtes. Un des critères de sélection des peupliers est désormais la brièveté de leur période feuillée, afin d'améliorer la croissance des cultures intercalaires.

\section{Les pratiques françaises et méditerranéennes}

Les cultures intercalaires dans des plantations d'arbres fruitiers sont fréquentes dans plusieurs régions françaises.

Les noyeraies du Dauphiné et du Périgord représentent probablement le plus grand exemple de cultures intercalaires d'Europe. La plupart des jeunes plantations sont entretenues par des cultures intercalaires, jusqu'à l'âge de 4 à 6 ans. Ces cultures comprennent surtout des céréales d'été telles le maïs (photo 1, p. 78) ou le sorgho, des céréales d'hiver (blé, orge), mais aussi des oléoprotéagineux (soja, colza, tournesol), du tabac, des plantes fourragères (luzerne), des plantes aromatiques telles que la lavande (photo 2, p. 78), des petits fruits (cassis, groseille), des arbres fruitiers (poiriers, pommiers, vigne), et des cultures de semence ou de fleurs (Liagre, 1993). Dans le Dauphiné, les noyeraies représentent une surface de 7300 ha. Près de 1500 ha sont l'objet de cultures intercalaires selon nos estimations, ce qui correspond à $80 \%$ des noyeraies de moins de 10 ans. Certains exploitants prolongent ces cultures intercalaires bien au-delà, et nous avons pu observer des parcelles de 15 ans avec de très belles cultures intercalaires. Notons que ces pratiques sont fortement déconseillées par les organismes de développement, et qu'elles ont été mises au point empiriquement par les exploitants. Le cahier des charges de l'appellation "noix de Grenoble" interdit même la culture intercalaire après l'âge de 4 ans des arbres. Une étude approfondie de ces pratiques de culture intercalaire permettrait de faire progresser rapidement les recherches en agroforesterie avec cultures intercalaires annuelles.

Les cultures peuvent avoir une influence très marquée sur la forme des arbres. Les maïs, souvent très hauts, peuvent engainer fortement les tiges et redresser les charpentières, lorsque la culture de maïs a été conduite de manière continue et très près des arbres (photo 1, p. 78). Une telle forme des arbres facilite la poursuite des opérations mécanisées entre les arbres. Cette influence peut conduire, dans le cas d'arbres cultivés pour le bois de leur bille de pied, à renforcer la fréquence d'apparition de fourches. Les agriculteurs préfèrent souvent des cultures moins hautes, telles le sorgho ou le soja, car elles permettent une meilleure ventilation du feuillage de l'arbre, et donc une sensibilité moindre aux maladies.

La vocation fruitière des arbres impose cependant des contraintes spécifiques pour la pratique des cultures intercalaires: sensibilité de l'arbre à des stress compétitifs au moment de l'induction florale (au mois de juin), nécessité de disparition de la culture intercalaire en septembre pour permettre la récolte mécanisée des noix, faible hauteur de la bille de pied qui ne facilite pas le passage des tracteurs entre les lignes d'arbres. On soulignera que ces trois contraintes très fortes, qui expliquent la fréquente disparition des cultures intercalaires à partir de la cinquième année, ne 


\section{DUPRAZ}



Photo 1

Culture associée noyers

à fruits - maïs dans la vallée de l'Isère.



Photo 2

Culture associée noyers

à fruits - lavandin dans

le Diois

(Drôme provençale).



Photo 3

Culture intercalaire de salades dans un verger de pêchers du Roussillon. Ici la proximité des arbres et des cultures impose une mécanisation au motoculteur.

Photos C DUPRAZ INRA Montpellier 


\section{L’agroligniculture}

sont pas présentes pour une agroforesterie avec des arbres à bois. Les exploitants rechignent à adopter les itinéraires techniques de la nuciculture moderne (fortes densités d'arbres, tailles basses en gobelet) précisément parce que de telles plantations empêchent la pratique de l'agroforesterie.

D'autres exemples d'agroforesterie avec arbres fruitiers et cultures intercalaires peuvent être observés en France. De nombreux vergers de pêchers du Roussillon sont valorisés par des cultures intercalaires hivernales maraîchères (photo $3, p .78$ ). II s'agit là d'une agroforesterie typique d'intensification de la valorisation d'un foncier restreint (vallées étroites), très comparable dans ses objectifs à l'agroforesterie chinoise. La technicité de ces pratiques force l'admiration. Cet exemple montre que l'agroforesterie peut permettre une valorisation de territoires irrigués, à très haute valeur foncière.

On retrouve de nombreux autres exemples d'agroforesteries similaires dans les pays méditerranéens: colture promiscue en Italie, vergers de chênes, d'oliviers, d'amandiers, de caroubier, d'arganiers avec cultures intercalaires de céréales dans les pays du pourtour méditerranéen. Ces pratiques sont très anciennes. Columelle, dans son monumental ouvrage "De re rustica", daté du premier siècle avant $J-C$, décrit ainsi la logique des cultures intercalaires de blé entre les oliviers : le blé est cultivé une année sur deux pour affaiblir la vigueur végétative des oliviers et favoriser ainsi la mise à fruit. Une oliveraie doit donc être divisée en deux parties, alternativement cultivées en céréales intercalaires, afin de produire une quantité élevée et régulière d'olives (Lelle et Gold, 1994).

Les peuplements clairs de chênes fruitiers de la péninsule lbérique méritent une mention spéciale. II s'agit de plantations à faible densité (30 à 60 tiges/ha) d'arbres sélectionnés pour leur production fruitière de glands doux, utilisés pour nourrir les ovins et les porcs, par pâturage automnal direct des glands tombés au sol. Appelés "dehesas" en Espagne et "montados" au Portugal, ces peuplements sont en fait des systèmes agrisylvicoles: des cultures intercalaires de céréales y étaient pratiquées tous les cinq à dix ans et avaient une fonction essentielle de suppression d'une strate arbustive envahissante. La raréfaction de ces mises en culture fait évoluer ces peuplements vers des formes sylvopastorales avec une strate arbustive délicate à contrôler.

On peut également observer dans certaines régions des pratiques de culture intercalaire de mais dans des plantations de Peuplier (vallée du Rhône, notamment), mais ces cultures sont extrêmement brèves (1 ou 2 ans) et les arbres sont installés à des densités forestières classiques.

\section{LA CONCEPTION DE NOUVELLES ASSOCIATIONS D'ARBRES ET DE GRANDES CULTURES}

\section{Les recherches nord-américaines}

Plusieurs expériences d'associations arbres - cultures annuelles ont été mises en place récemment au Canada et aux États-Unis.

Au Canada, les équipes de l'Université de Guelph, dans l'Ontario, suivent un dispositif expérimental qui totalise 26 ha de cultures associées chez cinq exploitants privés et 30 ha sur les terrains de I'Université (Gordon et Williams, 1991). Les essences d'arbres utilisées sont surtout le Noyer noir américain (Juglans nigra) et le Chêne rouge d'Amérique (Quercus rubra). Les cultures intercalaires sont le maïs, le soja et l'orge de printemps. Ces essais ont connu de nombreuses vicissitudes, avec notamment de redoutables sécheresses lors de leurs années d'installation en 1987 et 1988 . Les résultats des quatre premières années de culture intercalaire font apparaître que, pour des situations non irriguées, l'orge est une culture nettement moins favorable que les cultures d'été. En effet, sa précocité lui permet d'accomplir son cycle avant les arbres. Ceux-ci, au moment de leur débourrement, se trouvent donc confrontés à un compétiteur certes absent, mais qui laisse derrière lui un sol aux réserves en eau épuisées. Et surtout, la moisson précoce des céréales à paille laisse les arbres isolés au milieu d'un environnement sec, sans rugosité, ne les protégeant pas des effets 


\section{DUPRAZ}

du vent. Ces petits arbres sont alors soumis à une demande évaporative considérablement renforcée par la réflexion du sol et les advections d'air chaud (Baldy et al,, 1994). Dans une telle situation, des cultures d'été, a priori plus concurrentes puisque actives en même temps que les arbres, seraient préférables pour la croissance des arbres. On voit là que la recherche d'un décalage phénologique entre les arbres et les cultures associées, toujours favorable en ce qui concerne l'utilisation du rayonnement solaire, peut être dangereuse lorsque la compétition pour l'eau est importante.

Des expérimentations analogues sont en cours aux États-Unis (Garett et al., 1991), mais essentiellement avec le Noyer noir. La principale diftérence vient de ce que l'arbre est envisagé comme producteur de noix et de bois (noyer double-fin). Les cultures intercalaires sont poursuivies pendant 9 à 15 ans selon les sites, puis une prairie est installée sous les noyers, dont on prévoit la coupe à 60 ans. La production fourragère d'espèces à croissance printanière (Festuca arundinacea Schreb., Dactylis glomerata L., Phalaris arundinacea L.) est stimulée sous les arbres, avec une digestibilité in vitro améliorée par rapport à des prairies sans arbres. Les fourrages à croissance estivale sont au contraire moins performants sous les arbres. L'influence négative sur les cultures intercalaires de la juglone (5-hydroxy-1,4 naphtaquinone), cet exsudat foliaire et racinaire émis par le Noyer, semble tout à fait négligeable.

Pour les auteurs américains, le désherbage des associations d'arbres et de grandes cultures est une des questions clés. Un des essais de l'Université de Guelph a été fortement endommagé par des émanations de produits de désherbage épandus par temps venté sur une parcelle adjacente. Le désherbage mécanique de la bande non cultivée au pied des arbres est exclu par les différents auteurs, à cause des risques trop élevés de blessures aux arbres. Notons que les Américains ne connaissent pas encore l'usage des manchons de protection qui diminuent considérablement les risques de dégâts des phytocides de contact sur les petits arbres et permettent un repérage des plants qui facilite la mécanisation des entretiens.

Ces travaux expérimentaux ne semblent pas avoir encore eu d'impact auprès des exploitants agricoles, et les communications présentées à la troisième conférence sur l'agroforesterie en Amérique du Nord n'ont pas fait état de progrès en ce sens (Rule et al., 1993).

\section{Les recherches européennes}

À notre connaissance, les seuls dispositifs agroforestiers européens avec cultures annuelles intercalaires sont anglais. Les plus anciens datent de 1987 et 1988, dans le Buckinghamshire (Newman, 1991). Un premier essai associe des Peupliers (Populus deltoides $x$ trichocarpa) et du blé d'hiver. Les lignes d'arbres sont espacées de $14 \mathrm{~m}$, et les bandes non cultivées au pied des arbres sont couvertes d'un film plastique. En sixième année, les peupliers qui ont des accroissements annuels en hauteur d'environ $2 \mathrm{~m} / \mathrm{an}$ n'ont toujours pas d'effet significatif sur les rendements du blé (Newman, 1994). Les peupliers doivent être récoltés à 22 ans, et nous n'en sommes donc qu'au quart de la révolution. Un autre essai associe des Noyers et une rotation triennale (blé d'hiver, orge, colza). Après les quatre premières années, les rendements des cultures intercalaires n'étaient toujours pas affectés par la présence des arbres.

D'autres essais associent des feuillus précieux (Merisiers, Érables) avec des céréales, tels les essais de l'Université de Leeds. Ces essais récents n'ont pas encore fait l'objet de publications. La productivité des cultures intercalaires n'est pas affectée par la présence des arbres au cours des quatre premières années, alors que la croissance des arbres est légèrement freinée (communication personnelle Incoll). On peut regretter la très faible dimension de ces essais, qui exagère les effets de bordure.

II n'existe pour l'instant à notre connaissance aucune parcelle expérimentale agroforestière avec de grandes cultures intercalaires annuelles en France. Plusieurs expérimentations en Languedoc- 


\section{L'agroligniculture}

Roussillon correspondent à des plantations avec des cultures intercalaires fourragères pérennes: sainfoin, luzerne, fétuque élevée (Dupraz, 1994).

A partir de l'hiver 1994-95, une expérimentation doit être mise en place sur une cinquantaine d'hectares au nord de Montpellier, sur le domaine de Restinclières. Ce domaine appartient au Conseil général du département de l'Hérault et est exploité par deux agriculteurs. Des associations de différentes essences d'arbres à bois de qualité (Noyers hybrides, Cormiers) avec des cultures intercalaires de blé dur, colza et vigne y seront conduites dans les conditions d'une exploitation agricole réelle (GRAF, 1994). Les plantations agroforestières seront réalisées sur des sols profonds (agroforesterie de production à cycle rapide) et sur des sols superficiels (agroforesterie de protection et à vocation paysagère). Cette base expérimentale sera utilisée par divers partenaires de la recherche et du développement, pour l'étude du fonctionnement des associations arbres cultures d'une part, pour l'acquisition de références techniques et économiques d'autre part. Des initiatives similaires sont nécessaires dans les grandes régions céréalières françaises et pourraient être organisées en laboratoires sans murs ouverts à toute la communauté scientifique.

La faible influence des arbres sur les cultures au cours du premier quart de la révolution semble être généralisable. Cela signifie que la diminution de la production des cultures intercalaires est due essentiellement à la diminution des surfaces semées, et donc à la largeur des bandes non semées au pied des arbres. Inversement, l'effet des cultures sur les arbres est net si les bandes non cultivées sont étroites. Cette largeur de la bande non semée est un élément essentiel de l'optimisation d'itinéraires techniques de cultures associées modernes.

\section{Les recherches sur les cultures en couloir}

Une revue des pratiques et recherches en agroforesterie avec cultures annuelles intercalaires serait incomplète sans une présentation des techniques de cultures en couloir. Sous les tropiques, les recherches sur ces cultures sont très nombreuses et très avancées (voir par exemple, Singh et al., 1989 ; Karim et al., 1991).

La culture en couloir consiste à associer des arbres plantés en ligne et des cultures intercalaires annuelles (céréales, oléoprotéagineux). Les arbres sont utilisés comme une protection (brise-vent), mais surtout comme une source d'engrais: il s'agit toujours de légumineuses fixatrices d'azote, et les arbres sont recépés à plusieurs reprises au cours de la saison. Les produits de ces coupes (branches fines et feuilles) sont, soit utilisés comme fourrage, soit épandus entre les lignes de cultures annuelles. Dans ce cas, ils contribuent à la fertilisation des cultures (minéralisation), mais aussi à la lutte contre les adventices et à la limitation de l'évaporation du sol (effet de mulch). Ce sont les espèces des genres Leucaena et Gliricidia qui sont les plus utilisées dans ces systèmes de culture en milieu tropical.

La principale critique à l'encontre des cultures en couloir est leur gourmandise en travail. On remplace ainsi les intrants (engrais azotés, phytocides) par du travail, lequel est difficilement mécanisable. Ceci semble exclure de telles pratiques pour les agricultures des zones tempérées développées. Pourtant, certains auteurs ont cherché à imaginer des systèmes de cultures en couloir adaptées aux zones tempérées (Ssekabembe et Henderlong, 1991). Le Robinier (Robinia pseudoacacia L.) semble capable d'être le rival du Leucaena pour les zones tempérées (Barrett et Hanover, 1991), mais d'autres arbres fixateurs d'azote sont aussi envisagés (Alnus spp, Eleagnus spp).

La mécanisation de tels systèmes de cultures, avec utilisation d'une broyeuse (de type ensileuse) qui projetterait le broyat fertilisant sur la culture, n'a à notre connaissance pas été envisagée ni testée en zone tempérée. Avec le Robinier, la colonisation des terres cultivées par des drageons pourrait être un grave inconvénient. Mais les façons culturales suffiraient peut-être à limiter son expansion. Les données dont nous disposons permettent de calculer que des bandes de Robinier 


\section{DUPRAZ}

qui occuperaient $20 \%$ de la surface de la parcelle permettraient d'apporter environ 100 unités d'azote sur les $80 \%$ cultivés (par exemple en maïs), en deux passages de la broyeuse en juin et août (calcul effectué à partir des données de Barrett et Hanover, 1991).

\section{CONCLUSIONS}

En zone tempérée, peu d'associations d'arbres et de cultures intercalaires annuelles sont actueilement pratiquées à grande échelle dans le monde. C'est en Chine, avec l'agroforesterie du Paulownia associé au blé, que l'on trouve l'exemple le plus significatif, actuellement étendu sur plusieurs millions d'hectares. Cette agroforesterie a été mise au point récemment par les instituts chinois de recherche forestière. En France, les cultures intercalaires dans les vergers fruitiers (surtout les noyeraies) sont un exemple très évolué d'une agroforesterie mise au point empiriquement par les agriculteurs.

Hors de Chine, les recherches sur les associations d'arbres et de grandes cultures en zone tempérée sont actuellement limitées. Ce sont les universités des États-Unis, du Canada et de Grande-Bretagne qui ont mis en place quelques essais récemment.

En ouverture de la première conférence nord-américaine sur l'agroforesterie tempérée, J.B. Raintree exprimait au nom de la communauté agroforestière tropicale sa surprise et sa perplexité face à la transposition de la problématique agroforestière en zone tempérèe, avec des objectifs opposés à ceux assignés à l'agroforesterie tropicale: alors que tous les efforts en zone tropicale visent à améliorer la productivité du sol par l'association d'arbres et de cultures, des objectifs de réduction de la productivité et d'extensification étaient délibérément affichés pour une agroforesterie tempérée (Raintree, 1991).

Un consensus était cependant présent à cette époque : l'agroforesterie tempérée pouvait être une alternative intelligente de gestion des terroirs marginaux et extensifs (Gold et Hanover, 1987). Cette revue des différentes pratiques et recherches sur des associations d'arbres et de cultures intercalaires annuelles en zone tempérée nous a montré que au contraire certaines formes d'agroforesterie sont potentiellement capables de valoriser des terroirs agricoles riches, et très intensifiés. L'article "Le Chêne et le blé ..." (cf. p. 84) va nous permettre d'évaluer les perspectives d'adoption d'une telle agroforesterie dans les exploitations européennes de grande culture.



\section{BIBLIOGRAPHIE}

ACADÉMIE FORESTIÈRE CHINOISE (AFC). - Paulownia in China : cultivation and utilization / Zhu Zhaohua, Chao Ching-Ju, Lu Xin-Yu and Xiong Yao Gao Ed. . - Beijing, 1985. - $65 \mathrm{p}$.

BALDY (C.), DUPRAZ (C.), SCHILIZZI (S.). - Vers de nouvelles agroforesteries en climats tempérés et méditerranéens. Première partie : aspects agronomiques. - Cahiers Agricultures, n² 2, 1994, pp. 375-386.

BARRETT (R.P.), HANOVER (J.W.). - Robinia pseudoacacia: a possible temperate zone counterpart to Leucaena? In : Proceedings of the Second Conference on Agroforestry in North America / H.E. Garett Ed. . Columbia (Missouri), 1991. - pp. 27-41. 
CHIRKO (T.), GOLD (M.A.), JIANG (Jianping). - Paulownia Intercropping with winter Wheat on the Temperate North China Plain. In : Proceedings of the Third North American Agroforestry Conference. - Ames (Iowa), 1993. $-12 \mathrm{p}$.

DUPRAZ (C.). - Prospects for easing land tenure conflicts with agroforestry in Mediterranean France : a research approach for intercropped timber orchards. - Agroforestry Systerns, vol. 25, 1994, pp. 181-192.

DUPRAZ (C.), AUCLAIR (D.), GUITTON (J.-L.). - L'Agroforesterie néo-zélandaise. - Revue forestière française, Vol. XLIV, $n^{\circ} 6,1992$, pp. 523-538.

DUPRAZ (C.), GUITTON (J.-L.), RAPEY (H.), MONTARD (F.-X. de). - The treeshelter issue. In : Windbreaks and Agroforestry. - Viborg (Denmark), 1993. - pp. 106-111.

ÉTAIX (C.), LE HIR (I.). - Aperçu de réalisations agroforestières en République populaire de Chine. - Nancy ENGREF, 1988. - 153 p. (Rapport de voyage d'étude).

GARETT (H.E.), JONES (J.), KURTZ (W.B.), SLUSHER (J.P.). - Black walnut integrated Forestry-Farming ; a case history. In: Proceedings of the first Conference on Agroforestry in North America / P. Williams Ed. . University of Guelph, 1991. - pp. 15-28.

GOLD (M.A.), HANOVER (J.W.). - Agroforestry Systems for the Temperate Zone. - Agroforestry Systems, $n^{\circ} 5$, 1987, pp. 109-121.

GORDON (A.M.), WILLIAMS (P.A.). - Intercropping Valuable Hardwood Tree Species and Agricultural Crops in Southern Ontario: a research perspective. In: Proceedings of the first Conference on Agroforestry in North America / P. Williams Ed. . - University of Guelph, 1991. - pp. 29-55.

GRAF (Groupe de Recherches en AgroForesterie). - Avant-projet pour un aménagement agroforestier du domaine départemental de Restinclières / C. Dupraz Éd. . - Montpellier : INRA, 1994. - 33 p. + annexes.

HE (Qun). - Economic Evaluation of Intercropping with Paulownia - Optimisation and choice of intercropping models. In : Agroforestry Systems in China / Zhu Zhaohua, Cai Mantang, Wang Shiji and Jiang Youxu Ed. , CAF and IDRC Publishers, 1991. - pp. 77-88.

KARIM (A.B.), SAVILL (P.S.), RHODES (E.R.). - The effect of young Leucaena leucocephala (Lam) de Wit hedges on the growth and yield of maize, sweet potato and cowpea in an agroforestry system in Sierra Leone. Agroforestry Systems, $\mathrm{n}^{\circ}$ 16, 1991, pp. 203-211.

LELLE (M.A.), GOLD (M.A.). - Agroforestry Systems for Temperate Climates : Lessons from Roman Italy. - Forest and Conservation History, vol. 38, 1994, pp. 118-126.

LIAGRE (F.). - Les Pratiques de cultures intercalaires dans la noyeraie fruitière du Dauphiné. - Montpellier : ENGREF-INRA, 1993. - 80 p. + annexes (Mémoire de mastère en Sciences forestières).

LIU (Naizhuang), XIONG (Qinxue). - Solar radiation Distribution in Intercropped Fields with Paulownia. In : Agroforestry Systems in China / Zhu Zhaohua, Cai Mantang, Wang Shiji and Jiang Youxu Ed. . - CAF and IDRC Publishers, 1991. - pp. 66-76.

NEWMAN (S.M.). - An outline comparison of approaches to silvoarable research and development with fast growing trees in India, China, and the UK with emphasis on intercropping with wheat. Biodiversity International Limited. - Agroforestry Forum, vol. 5, $\mathrm{n}^{\circ} 2$, 1994, pp. 29-31.

NEWMAN (S.M.), WAINWRIGHT (J.), OLIVER (P.N.), ACWORTH (J.M.). - Walnut agroforestry in the UK : Research (1900-1991) assessed in relation to experience in other countries. In: Proceadings of the 2 nd Conference on Agroforestry in North America. - Springfield (Missouri), août 1991. - pp. 74-94.

RAINTREE (J.B.). - Agroforestry in North America : the next generation. In : Proceedings of the first Conference on Agroforestry in North America / P. Williams Ed. . - University of Guelph, 1991. - pp. 1-14.

RULE (L.C.), COLLETTI (J.P.), LIU (T.P.), JUNGST (S.), MIZE (C.), SCHULTZ (R.C.). - Agroforestry in the Midwestern United States. In : Opportunities for Agroforestry in the Temperate Zone Worldwide/R. Schultz et J. Colletti Ed. . - Universitè de l'lowa, 1994, à paraître.

SAINT-PIERRE (C.). - Cultures associées en Chine : une ètude de cas sur la diffusion des innovations agricoles. 11 p. - À paraître dans Les Cahiers de la Recharche-Développernent, 1995.

SINGH (R.P.), ONG (C.K.), SAHARAN (N.). - Above and below ground interactions in alley cropping in semi-arid India. - Agroforestry Systems, vol. 9, 1989, pp. 259-274.

SSEKABEMBE (C.K.), HENDERLONG (P.R.). - Below-ground interactions in alley cropping ; Appraisal of tirst-year observations on maize grown in black locust alleys. In: Proceedings of the Second Conference on Agroforestry in North America / H.E. Garett Ed. . - Columbia (Missouri), 1991. - pp. 58-73.

TEJWANI (K.G.). - Agroforestry in hill regions of India. In: Agroforestry for rural needs / D.K. Khurana et P.K. Khosla Ed. . - Solan (Inde) : Indian Society of Tree Scientisfs, 1987. - pp. 389-407.

TEWARI (D.N.). - Poplar. - Surya Publications (India), 1993. - $321 \mathrm{p}$.

WU (Yunying), DALMACIO (R.V.). - Energy Balance, Water Use and Wheat Yield in a Paulownia-Wheat Intercropped Field. In : Agroforestry Systems in China / Zhu Zhaohua, Cai Mantang, Wang Shiji and Jiang Youxu Ed. . - CAF and IDRC Publishers, 1991. - pp. 54-65.

YOU (Xiuling). - Mixed cropping with trees in Ancient China. In : Agroforestry Systems in China / Zhu Zhaohua, Cai Mantang, Wang Shiji and Jiang Youxu Ed. . - CAF and IDRC Publishers, 1991. - pp. 8-9.

$\mathrm{ZHU}$ (Zhaohua). - Evaluation and model optimisation of Paulownia Intercropping System - A project summary reporf. In : Agroforestry Systems in China / Zhu Zhaohua, Cai Mantang, Wang Shiji and Jiang Youxu Ed. CAF and IDRC Publishers, 1991. - pp. 30-43.

ZHU (Zhaohua), FU (Maoyi), SASTRY (C.B.). - Agroforestry in China, an overview. In : Agroforestry Systems in China / Zhu Zhaohua, Cai Mantang. Wang Shiji and Jiang Youxu Ed. . - CAF and IDRC Publishers, 1991. pp. $2-7$.

ZHU (Zhaohua), LU (Xinyu), XIONG (Yaoguo). - Paulownia. - The Chinese Academy of Forestry, 1991, - 26 p. 